\title{
Marine-derived compound-A suppresses zinc-enhanced pro-inflammatory M1 phenotype of microglia via inhibition of ROS generation
}

\author{
Youichirou Higashi, Takaaki Aratake, Takahiro Shimizu, Shogo Shimizu, Yusuke Ueba, \\ Tomoya Hamada, Zou Suo, Masaki Yamamoto, Yoshiki Nagao, Motoaki Saito
}

Pharmacology, Kochi Medical School, Kochi University, Japan

[BACKGROUND] Under ischemic conditions, microglia exhibit two opposite activation states (inflammatory M1 and anti-inflammatory M2 activation). Recent studies have indicated that increase in the M1 microglia exacerbates postischemic brain damage. We have previously demonstrated that ischemia-induced extracellular zinc release from neurons primes microglia to enhance production of pro-inflammatory cytokines via reactive oxygen species (ROS) generation in response M1 stimuli. Here, we evaluate the effect of marine-derived compound-A (MD-A) on the zinc-enhanced inflammatory M1 phenotype of microglia.

[METHODS] M1 activation of microglia from neonatal mice was induced by $1 \mathrm{ng} / \mathrm{mL}$ lipopolysaccharide (LPS) after 60 microM $\mathrm{ZnCl} 2$ pretreatment in the presence of $30-300 \mathrm{ng} / \mathrm{mL} \mathrm{MD}-\mathrm{A}$, and pro-inflammatory cytokines secretion were assessed by ELISA. In order to detect ROS generation, DHE, a general probe for ROS, was added to microglial culture before $\mathrm{ZnCl} 2$ treatment in the presence of $\mathrm{MD}-\mathrm{A}$. Young adult mice were subjected to transient ischemia 5 min after injection (i.c.v.) of MD-A. The expression of inflammatory cytokines and M1 markers were examined by real-time PCR and immunohistochemistry, respectively.

[RESULTS] Treatment of microglia with MD-A resulted in a dose-dependent attenuation of the zinc-enhanced proinflammatory cytokines secretion from LPS-stimulated microglia. DHE staining was markedly increased in microglia treated with zinc, which was suppressed by MD-A. I.c.v. pre-injection of MD-A suppressed ischemia-induced increase in the expression of pro-inflammatory cytokines and M1 marker.

[CONCLUSION] These results demonstrate that MD-A prevents extracellular zinc-enhanced pro-inflammatory cytokines secretion from M1 microglia by suppressing microglial ROS generation, which suggests that MD-A may be a promising therapeutic agent for stroke. 